\title{
Erratum: Excitation effect of $d$ electrons on the electronic energy loss of energetic protons colliding with a Zn atom [Phys. Rev. A 101, 062705 (2020)]
}

\author{
Xu-Dong Zhao, Fei Mao®, Sheng-An Tang, Cong-Zhang Gao, Feng Wang, and Feng-Shou Zhang \\ (Received 11 December 2020; published 29 December 2020)
}

DOI: 10.1103/PhysRevA.102.069905

The units of experimental data [1] (cited as Ref. [38] in original paper) and the SRIM data in Fig. 10 of our paper are incorrect. We provide below the corrected version of Fig. 10; the caption from the original article is also included but it has not changed.

It can be seen from the figure that our calculated stopping cross section (SCS) underestimates the experimental SCS [1] and the SRIM data beyond the measured stopping maximum. However, these changes do not affect the conclusion of the paper.

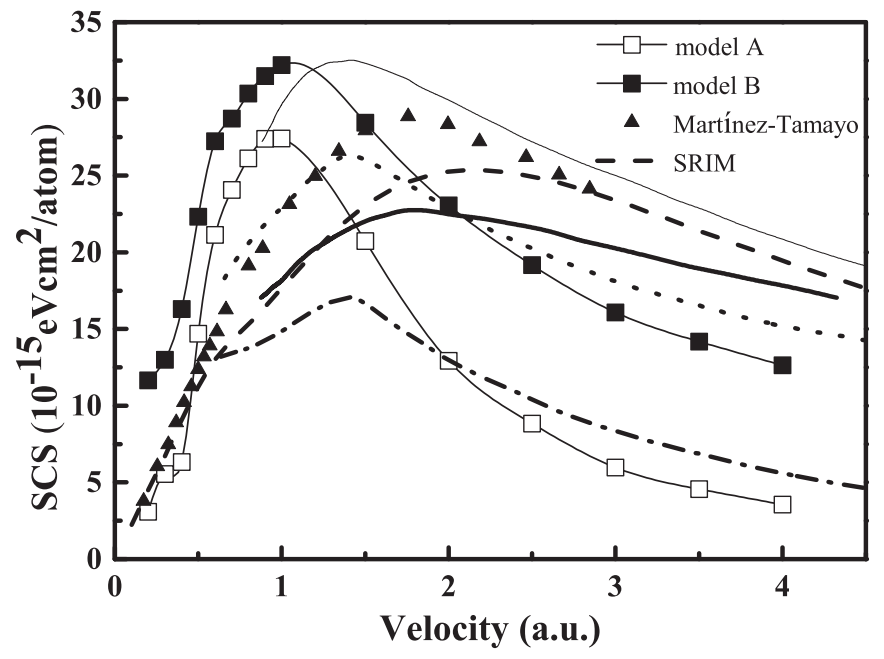

FIG. 10. Stopping cross section for proton colliding with atomic zinc as a function of the velocity. Open and solid squares indicate the results obtained from models $\mathrm{A}\left(\left[\mathrm{Ar} 3 d^{10}\right] 4 s^{2}\right)$ and $\mathrm{B}\left([\mathrm{Ar}] 3 d^{10} 4 s^{2}\right)$, respectively. The dotted and dashed-dot lines indicate the contributions of $3 d+4 s$ and $4 s$ electron excitation to the stopping cross section of zinc [39], respectively. The thick and thin lines indicate the experimental data of solid and gas phase zinc [39], respectively. The triangles indicate the experimental measurements of bulk zinc from Ref. [38]. The dashed line indicates the SRIM data.

[1] G. Martínez-Tamayo, J. C. Eckardt, G. H. Lantschner, and N. R. Arista, Phys. Rev. A 54, 3131 (1996). 